\title{
Isotopically modified Ge detectors for GERDA: from production to operation
}

\author{
D. Budjáš ${ }^{i}$, M. Agostini ${ }^{i}$, L. Baudis ${ }^{l}$, E. Bellotti ${ }^{e, f}$, L. Bezrukov ${ }^{g}$, R. Brugnera ${ }^{j}$, C.

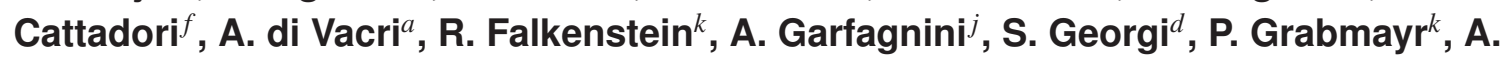 \\ Hegai $^{k}$, S. Hemmer ${ }^{j}$, M. Hult' ${ }^{c}$, J. Janicskó Csáthy ${ }^{i}$, V. Kornoukhov ${ }^{g, h}$, B. Lehnert ${ }^{b}, \mathbf{A}$. \\ Lubashevskiy $^{d}$, S. Nisi ${ }^{a}$, G. Pivato ${ }^{j}$, S. Schönert ${ }^{i}$, M. Tarka ${ }^{l}$, and K. von Sturm ${ }^{k}$ \\ ${ }^{a}$ INFN Laboratori Nazionali del Gran Sasso LNGS, Assergi, Italy \\ ${ }^{b}$ Institut für Kern- und Teilchenphysik Technische Universität Dresden, Dresden, Germany \\ ${ }^{c}$ Institute for Reference Materials and Measurements, Geel, Belgium \\ ${ }^{d}$ Max Planck Institut für Kernphysik, Heidelberg, Germany \\ e Università di Milano Bicocca, Milano, Italy \\ ${ }^{f}$ INFN Milano Bicocca, Milano, Italy \\ ${ }^{g}$ Institute for Nuclear Research of the Russian Academy of Sciences, Moscow, Russia \\ ${ }^{h}$ Institute for Theoretical and Experimental Physics, Moscow, Russia \\ ${ }^{i}$ Physik Department E15 and Excellence Cluster Universe, T.U. München, Germany \\ ${ }^{j}$ Dipartimento di Fisica e Astronomia dell'Università di Padova e INFN, Padova, Italy \\ ${ }^{k}$ Physikalisches Institut, Eberhard Karls Universität Tübingen, Tübingen, Germany \\ ${ }^{l}$ Physik Institut der Universität Zürich, Zürich, Switzerland \\ E-mail: grabmayreuni-tuebingen.de
}

ABSTRACT: The GERDA experiment searches for the neutrinoless double beta $(0 v \beta \beta)$ decay of ${ }^{76} \mathrm{Ge}$ using high-purity germanium detectors made of material enriched in ${ }^{76} \mathrm{Ge}$. For Phase II of the experiment a sensitivity for the half life $T_{1 / 2}^{0 v} \sim 2 \cdot 10^{26} \mathrm{yr}$ is envisioned. Modified Broad Energy Germanium detectors (BEGe) with thick $\mathrm{n}^{+}$electrodes provide the capability to efficiently identify and reject background events, while keeping a large acceptance for the $0 v \beta \beta$-decay signal through novel pulse-shape discrimination (PSD) techniques. The viability of producing thickwindow BEGe-type detectors for the GERDA experiment is demonstrated by testing all the production steps from the procurement of isotopically modified germanium up to working BEGe detectors. Comprehensive testing of the spectroscopic as well as PSD performance of the GERDA Phase II prototype BEGe detectors proved that the properties of these detectors are identical to those produced previously from natural germanium material following the standard production line of the manufacturer.

Furthermore, the production of BEGe detectors from a limited amount of isotopically modified germanium served to optimize the production, in order to maximize the overall detector mass yield. The results of this test campaign provided direct input for the subsequent production of the enriched germanium detectors.

KEYWORDS: Double beta decay; HPGe detectors; detector production. 


\section{Contents}

1. Introduction [1]

2. Procurement and production 20

2.1 Material procurement and isotopic analysis 3

2.2 Crystal production 3

2.3 Detector manufacturing

2.4 The time line 5]

3. Detector characterization 5

3.1] Operational characteristics of the detectors

3.1.1 The depletion voltage 6

3.1.2 The dead layer 6

3.1.3 The active volume

3.1.4 Determination of uniformity from surface scans 7

3.1.5 Determination of active lengths by surface scans 8

3.1.6 Summary of active and dead volume determinations 8

3.2 Tests of the spectroscopic performance 9

3.2.1 Energy resolution 9

3.2.2 Pulse shape discrimination

3.2.3 Long-term stability 13

田. Conclusions 13

\section{Introduction}

GERDA [ [1], 2] is an experiment located at Laboratori Nazionali del Gran Sasso (LNGS) of INFN that searches for the neutrinoless double beta $(0 v \beta \beta)$ decay employing high-purity germanium (HPGe) detectors enriched in the isotope ${ }^{76} \mathrm{Ge}$. The bare detectors are operated in liquid argon in order to minimize the background from radioactive isotopes in materials near the detectors. The first phase of the experiment is employing reprocessed detectors $(18 \mathrm{~kg}$ total mass, enriched to $86 \%$ in ${ }^{76} \mathrm{Ge}$ ) from the past experiments HDM and IGEX [3], 田]. GerDA aims at a reduction of the background in the region of interest at $Q_{\beta \beta}=2039 \mathrm{keV}$ to a level of $10^{-2} \mathrm{cts} /(\mathrm{keV} \cdot \mathrm{kg} \cdot \mathrm{yr})$, which is about a factor of 10 lower than previous state-of-art. So far, a background index (BI) of $\sim 2 \cdot 10^{-2} \mathrm{cts} /(\mathrm{keV} \cdot \mathrm{kg} \cdot \mathrm{yr})$ has been reached [2] $]$. In the absence of a signal and given the current BI, GERDA expects to set $90 \%$ probability lower limits of $T_{1 / 2}>1.9 \cdot 10^{25} \mathrm{yr}$ for an exposure of $20 \mathrm{~kg} \cdot \mathrm{yr}$ in Phase I [2] to scrutinize the claim [5]. In order to improve the half-life sensitivity by a factor 10 in Phase II of the experiment, $20 \mathrm{~kg}$ of new detectors from enriched germanium $\left({ }^{\mathrm{enr}} \mathrm{Ge}\right)$ 
will be added to reach the proposed exposure of $100 \mathrm{~kg} \cdot \mathrm{yr}$ within reasonable time. Simultaneously, the $\mathrm{BI}$ is expected to be reduced to $10^{-3} \mathrm{cts} /(\mathrm{keV} \cdot \mathrm{kg} \cdot \mathrm{yr})$ or below [1].

Apart from improved shielding, active background suppression techniques will be employed to reach the envisioned BI of $\lesssim 10^{-3} \mathrm{cts} /(\mathrm{keV} \cdot \mathrm{kg} \cdot \mathrm{yr})$. The measures comprise the instrumentation of the liquid argon and new enriched germanium detectors. The chosen detector type for Phase II is a modified thick window Broad Energy Germanium (BEGe) detector manufactured by Canberra Olen, Belgium [6]. Their proper functioning in LAr has been demonstrated [Z]. In comparison with the presently used coaxial germanium detectors, BEGe detectors have lower noise which translates into better energy resolution. Furthermore, their characteristic internal electric field distribution allows the identification of $0 v \beta \beta$-decay like signals and rejection of background signals with higher sensitivity and robustness using a novel technique of pulse-shape discrimination (PSD) [\&, ㅈ].

Compared to coaxial HPGe detectors used in GERDA Phase I and past $0 v \beta \beta$ experiments [3], 团, the BEGe detector design imposes tighter constraints on the impurity and defect concentrations in the germanium crystal material. These requirements arise due to the non-coaxial electrode arrangement in BEGe detectors and the resulting electric field profile; these are more demanding to achieve complete charge collection within the detector volume.

To demonstrate that working BEGe detectors with the desired properties can be produced from the procured ${ }^{\text {enr }} \mathrm{Ge}$ material, a comprehensive validation was performed. The tests of the production chain started from procurement of isotopically modified germanium material of the same chemical history as the already available ${ }^{\text {enr }} \mathrm{Ge}$, i.e. the leftovers from the enrichment process, depleted in ${ }^{76} \mathrm{Ge}$, that is named ${ }^{\text {dep }} \mathrm{Ge}$. Further steps included the purification, crystal pulling and diode production. The tests finished with spectroscopic and PSD performance characterizations of the manufactured detectors.

A second goal was the optimization of the production processes in order to maximize the mass yield of functional detectors from the available amount of ${ }^{\mathrm{enr}} \mathrm{Ge}$ material. These improvements were directly implemented into the production process of the GERDA Phase II ${ }^{\text {enr }} \mathrm{Ge}$ detectors. The data obtained with the characterization of the ${ }^{\mathrm{dep}} \mathrm{Ge}$ BEGes provide an important reference for assessing the performance of new ${ }^{\text {enr }}$ Ge BEGes.

The various steps of the production chain are described in the following section [. Section 3 reports the results of the detector characterization.

\section{Procurement and production}

The supply chain starts with the procurement of isotopically modified germanium in the form of $\mathrm{GeO}_{2}$. The isotopic composition is then confirmed by different types of material analyses performed at different laboratories. The production process continues by reduction of the oxide to its metallic form and subsequent purification via zone refinement. This is followed by crystal pulling, the determination of electrically active impurity concentrations in the crystal volume, and selection and cutting of slices useful for detector production. Detectors are fabricated by transforming the germanium crystal slices into junctions that are then mounted into vacuum cryostats for tests of their properties. 


\subsection{Material procurement and isotopic analysis}

In total, $34 \mathrm{~kg}$ of ${ }^{\mathrm{dep}} \mathrm{GeO}_{2}$ material was acquired in early 2009 from ECP Zelenogorsk, Russia [10]. This material was a by-product from the previous enrichment process for GERDA and was chemically processed identically as the enriched germanium material. Given the higher abundance of ${ }^{\text {dep }} \mathrm{Ge}$ after the enrichment process with respect to the ${ }^{\mathrm{enr}} \mathrm{Ge}$, the cost per mass of ${ }^{\text {dep }} \mathrm{Ge}$ is substantially lower. The only physical difference of the chemical identical material, is the isotopic composition and in particular the abundance of the isotope ${ }^{76} \mathrm{Ge}$.

Several $1 \mathrm{~g}$ sized samples of the $\mathrm{GeO}_{2}$ material were used for isotopic analysis via inductivelycoupled plasma mass spectrometry (ICPMS) and neutron activation analysis (NAA). The combined results for the isotopic abundance $f_{G e}$, i.e. the relative number of nuclei, are shown in Table 1 . The standard deviation is dominated by the different results from the various methods rather than by the individual statistical uncertainties of the measurements. An isotopic analysis of the material after completing the full production chain is envisioned. Such a test could detect possible admixtures of natural germanium $\left({ }^{\text {nat }} \mathrm{Ge}\right)$ during the production processes. In order to maximize the sensitivity for such tests, the isotopic abundance of ${ }^{76} \mathrm{Ge}$ in the procured material was required to be $<1 \%$ from the beginning.

Table 1. Isotopic abundance $f_{G e}$ of the depleted $\mathrm{GeO}_{2}$ samples, averaged over all measurements performed at LNGS (ICPMS), INR RAS, Moscow (ICPMS), University of Tübingen (NAA, with sample irradiation at FRM II Munich) and IRMM, Geel, Belgium (NAA, with sample irradiation at SCK•CEN, Mol, Belgium).

\begin{tabular}{c|ccccc} 
isotope & 70 & 72 & 73 & 74 & 76 \\
\hline$f_{G e}$ & $0.223(8)$ & $0.300(4)$ & $0.083(2)$ & $0.388(6)$ & $0.006(2)$
\end{tabular}

\subsection{Crystal production}

After taking samples for ICPMS the input was $33.84 \mathrm{~kg}$ of $\mathrm{GeO}_{2}$ material that was reduced to its metallic form and purified via zone refinement at PPM Pure Metals in Langelsheim, Germany [11]. This process yielded $21.49 \mathrm{~kg}$ of $6 \mathrm{~N}$ grade germanium with specific resistivity $\rho_{\text {spec }}>50 \Omega \mathrm{cm}$ and $\sim 1.37 \mathrm{~kg}$ beyond this specification. Using the specified abundances and the atomic mass $M_{A}=72.44$ the input mass amounts to $23.47 \mathrm{~kg}$ metallic germanium. Thus, the purification process was performed with an efficiency of $91.6 \%$.

Crystal pulling and detector slice production was performed at Canberra Oak Ridge, USA [12]. The manufacturer modified its production process with the objective to maximize the total mass yield of final detectors for a fixed amount of starting germanium material. In total four p-type crystals were produced from which 14 detector slices were cut with a total mass of $10.0 \mathrm{~kg}$. This corresponds to a yield of $46.5 \%$ with respect to the starting $21.49 \mathrm{~kg}$ of $6 \mathrm{~N}$ depleted germanium material. Given the exploratory goal of this production test, also non-standard crystal slice dimensions and shapes were tested. Moreover, slices close to the seed-end and tail-end side of the crystal were investigated, to test whether working detectors can be produced. The number of test and inspection slices, usually $1 \mathrm{~mm}$ thick, was minimized. 

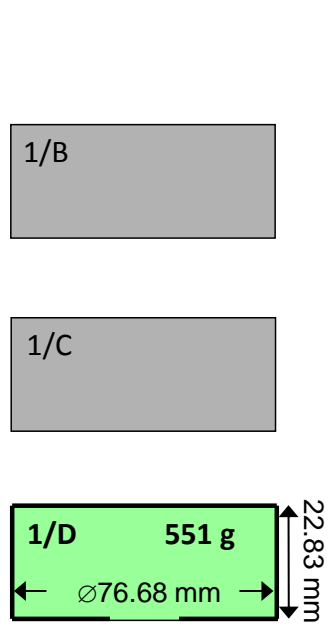

2

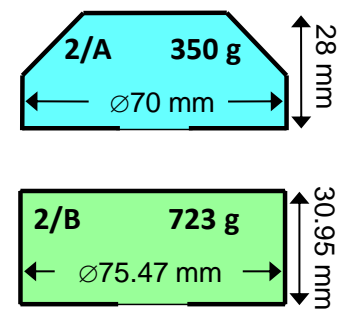

$2 / \mathrm{C}$

$2 / D$
3
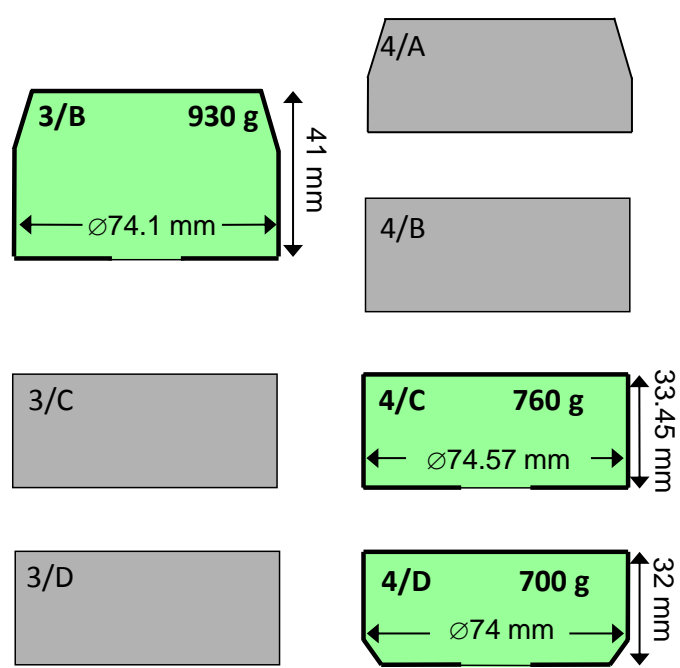

Figure 1. Summary plot of the dep Ge crystals and their slices. The orientation of the crystals is such that the seed end is on the top. The slices processed into detectors are shown in green, and the one slice which was turned into a functional diode, but not mounted into a vacuum cryostat is shown in blue. The thicker border line of the processed slices indicates the approximate extent of the Li-infused layer. The masses of the slices are given in $\mathrm{g}$.

Prior to defining the dimension of each detector slice, a full electrical field calculation was carried out for the conceived detector geometry, redundantly by the manufacturer and by us. For the latter the method of field calculations is summarized in Ref. [9]. Design criteria required that the depletion voltage should not exceed $4 \mathrm{kV}$ and that all regions inside the detector volume should have sufficient strong electrical fields to ensure full charge collection. The diameter of the final slices vary from $72 \mathrm{~mm}$ to $78 \mathrm{~mm}$, the height from $23 \mathrm{~mm}$ to $41 \mathrm{~mm}$ and the mass from $350 \mathrm{~g}$ to $930 \mathrm{~g}$. A schematic view of the crystal slices is depicted in Fig. 1. The detector slice identification used in this report follows the order of crystal production and a letter corresponding to one of maximal four slices cut from each crystal; with A being the seed-end slice and D the tail-end slice.

\subsection{Detector manufacturing}

The detector manufacturing was finalized at Canberra Olen, Belgium [ [6], by creating the Li-infused $\mathrm{n}^{+}$electrode covering most of the surface, a B-implanted small-area $\mathrm{p}^{+}$electrode located in the middle of the bottom flat face of the cylindrical slice and a circular non-conductive groove separating the two electrodes. The modified BEGe detectors for GERDA differ from the standard production version [13]. The latter have a thin entrance window on the top flat surface to increase the sensitivity to low energy photons, while the GERDA version has a $0.4 \mathrm{~mm}$ to $0.8 \mathrm{~mm}$ Li-infused $\mathrm{n}^{+}$electrode also on this part of the detector surface.

The detectors were mounted in standard vacuum cryostats, equipped with Canberra 2002CSL charge sensitive preamplifiers. Five crystal slices were transformed into detectors with at least 
one slice taken from each crystal. The mass loss turning a crystal slice into a diode is usually of the order of ten grams and thus negligible. However, slice 2/A was turned into a working diode only after removal of approximately $160 \mathrm{~g}$ of material from the seed-end side. This diode was not mounted in a vacuum cryostat because of its chamfered shape, which is incompatible with standard detector holders.

The mass yield of crystal slices can be further increased to approximately $55 \%$ employing twice the initial mass, as employed in the production of enriched detectors. Residual crystal pieces, that are not adequate for detector production, can return into the zone refinement or crystal pulling process. Moreover, it is estimated that a large fraction of kerf and grinding losses can be recovered, chemically reprocessed and returned to the zone refinement and crystal pulling process.

\subsection{The time line}

The procurement and production has been achieved in a rather short time. It proved rather helpful that: $(i)$ negotiations on buying isotopically modified material form ECP started in Moscow on February 26, 2009. The material arrived in Tübingen on April 30. Within one week 40 samples were analyzed regarding the isotopic composition by four institutes. (ii) Zone refinement at PPM Pure Metals was accomplished by June 2009. (iii) The $6 \mathrm{~N}$ material arrived at Oak Ridge on August 7. (iv) The first shipment of three detector grade slices left by plane for Olen on December 16, 2009. (v) The first two detectors were ready on March 19, 2010. (vi) The second batch of two detector slices was sent to Olen on June 15, 2010. (vii) The last shipment occurred on January 11, 2011.

The larger periods between the shipments was owing to intermediate testing at Laboratori Nazionali del Gran Sasso. Thus, within less than 2 years the production chain could be setup and verified. Having understood the details, the enriched BEGe detectors could be produced even faster. The main difference concerns a more carefully shielded transport to avoid activation of the enriched material.

\section{Detector characterization}

The five detectors produced underwent a comprehensive testing campaign to characterize their charge collection, spectroscopic and PSD performance, as well as long-term stability. An analog data acquisition (DAQ) chain consisting of a standard spectroscopy amplifier set to $10 \mu$ s shaping time and a multi-channel analyzer was used for the spectroscopic measurements. Digital recording of pulse shapes for subsequent off-line PSD analysis was accomplished using a DAQ system consisting of a non-shaping amplifier and a Struck SIS 3301 14-bit flash analog-digital converter with a read-out speed of $10^{8}$ samples per second similar to the one used for the GERDA acquisition at LNGS [2]. The recorded pulse-shape data were processed and analyzed using GELATIO [14], a GERDA software framework for advanced data analysis and digital signal processing.

Four out of five detectors show a rather similar behavior resulting in the same parameters comparable to the standard detector. Only detector 1/D exhibits a reduced performance, the origin of which is not fully clarified. It was the last detector delivered and was tested separately. Various conjectures will be discussed in the following, e.g. noise contributions or inhomogeneous impurity distribution. However, none of these hypotheses can be finally rejected or proven. 


\subsection{Operational characteristics of the detectors}

The first set of measurements was carried out to investigate the operational characteristics of the detectors. This included the determination of: (i) the depletion voltage, (ii) the dead layer, (iii) the active volume and other active dimensions, and (iv) the uniformity of charge collection along detector surfaces.

The tests were iterated to ensure that a stable optimum for the parameters had been reached. Table $[$ summarizes the main results along with the equivalent results for a reference detector a BEGe from standard Canberra production made of natural germanium, with $81 \mathrm{~mm}$ diameter, $32 \mathrm{~mm}$ thickness and a mass of $878 \mathrm{~g}$ [[] $]$. The particulars of the individual measurements are discussed below. More details about the dead layer and active volume measurements can be found in chapter 8 of Ref. [15].

Particularly useful are scans along the surfaces of the detectors. A ${ }^{241} \mathrm{Am}$ source was collimated to provide a $59 \mathrm{keV}$ photon beam within a spot with a diameter of $\sim 1 \mathrm{~mm}$ on the detector surface. Due to the collimation, only a small fraction of emitted photons reach the detector. For ease of comparison between different detectors and between results and Monte Carlo predictions the normalized counts rates $R_{n}$, namely detected photons over emitted photons, are plotted as function of position. The activity of the sources is known to $1.5 \%$ uncertainty. The intensity distributions $R_{n}$ will be compared with Monte Carlo (MC) simulations as shown in Fig. 2].

\subsubsection{The depletion voltage}

The reverse-bias voltage at which the detector volume is maximally depleted of free charge carriers, referred to as the depletion voltage $V_{d e p}$, was determined first. The corresponding volume in the detector is called 'active volume' $V_{a c t}$, while the remaining part, in general the surface with the 'dead layer' and electrodes, remains inactive. The depletion voltage is found by irradiating the detector with a ${ }^{60} \mathrm{Co}$ source while scanning the bias high-voltage range and determining the minimal voltage at which the spectroscopic characteristics (rate, width and position of $\gamma$ lines) are unchanged. Table $\mathrm{Z}$ also includes the operational bias voltage $V_{o p}$ as recommended by the manufacturer. $V_{o p}$ is a conservative value above the full depletion clearing the active volume free of charge carriers. The excess of $V_{o p}$ over $V_{d e p}$ determined by the bias voltage scan is typically $1 \mathrm{kV}$.

\subsubsection{The dead layer}

To determine the thickness of the Li-infused $\mathrm{n}^{+}$surface dead layer on the top area of the detector, the intensities of ${ }^{241} \mathrm{Am}$ and ${ }^{133} \mathrm{Ba}$ low-energy $\gamma$ lines are compared with Monte Carlo (MC) simulations as described in ref. [15, 16, 17, [18]. The values of the measured dead layer, $d_{d}$, are collected in Table 2]. The uncertainties include, besides the statistical and fit uncertainties, also a measure of the systematic uncertainty of the determination methods, expressed as the standard deviation of the results obtained with the ${ }^{241} \mathrm{Am}$ and ${ }^{133} \mathrm{Ba}$ methods. Knowing the thickness $d_{d}$ the involved mass $m_{d}$ can be calculated. A first estimate for the active mass fraction from this geometrical consideration can made: $f_{\text {geo }}=\left(m_{t o t}-m_{d}\right) / m_{t o t}$. Values in the range $(91-96) \%$ are obtained from this procedure as shown in Table 2 . 

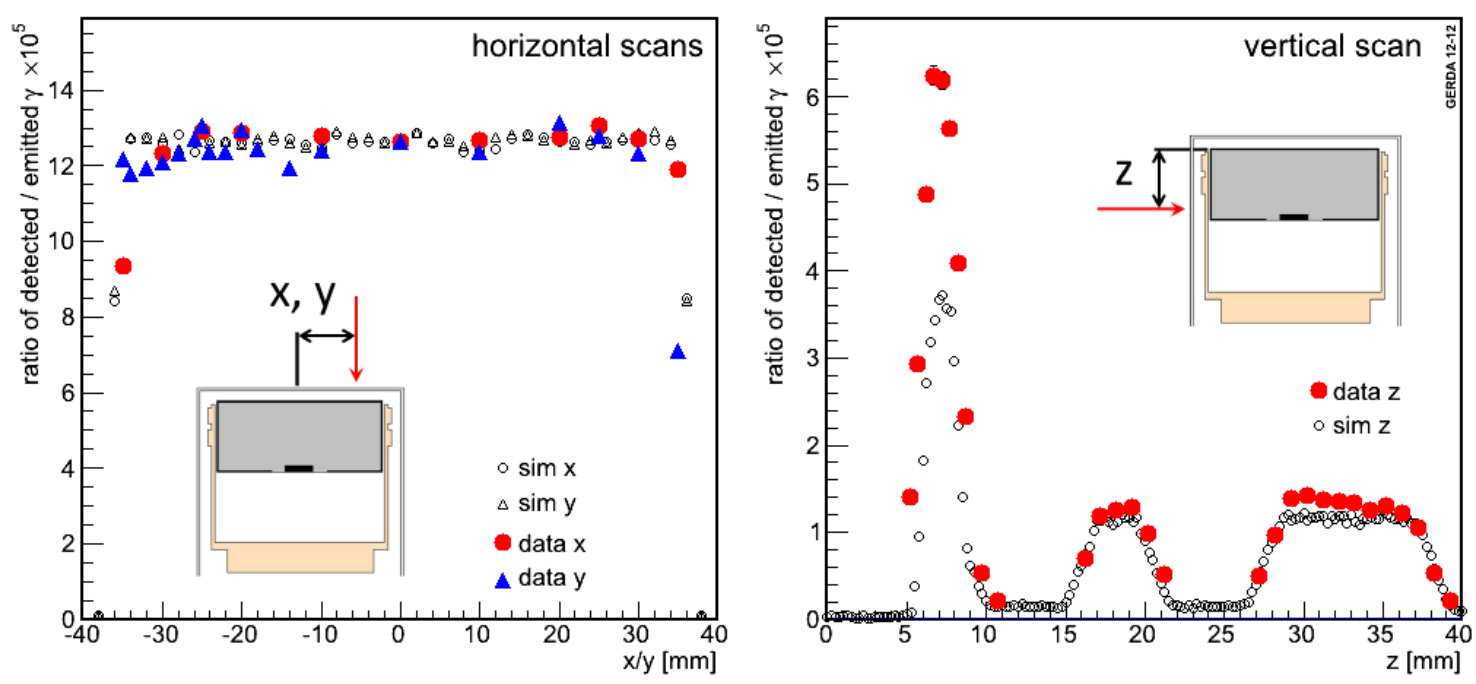

Figure 2. Normalized intensities $R_{n}$ for the $59 \mathrm{keV}$ peak of the ${ }^{241}$ Am source compared to MC predictions. The respective insets show the detector vacuum housings and holder cross sections with the beam position and direction indicated. Left: $R_{x, y}$ from the horizontal scans in $x$ - and $y$-direction for the detector 4/D. Right: $R_{z}$ from a vertical scan along the side surface of detector $4 / \mathrm{C}$.

\subsubsection{The active volume}

The active volume $V_{a c t}$ is determined by irradiating the detector with ${ }^{60} \mathrm{Co}$ and reproducing the $1332.5 \mathrm{keV} \gamma$ line detection efficiency with MC simulation while assuming that the only inactive part of the detector volume is the Li-infused $\mathrm{n}^{+}$surface dead layer (c.f. ref. [15, [16, 117]). The uncertainty is dominated by the uncertainty of the source intensity and of its position with respect to the detector. In determining the active fraction of the detector volume $f_{a v}$, a small contribution to its uncertainty arises also from the uncertainties of the outer geometrical dimensions provided by the manufacturer. Comparing the results in Table , the nice agreement of $f_{a v}$ with $f_{\text {geo }}$ is observed. Only detector 1/D shows a strong deviation that can not be explained so far.

\subsubsection{Determination of uniformity from surface scans}

The characterization of the uniformity of charge collection along the detector surfaces is performed by a scan, that observes the variation of spectroscopic parameters (peak position and energy resolution) along the scanning points. The variation of these parameters was found to be smaller than the random fluctuations caused by electronic disturbances in the DAQ setup: typically below $\pm 0.1 \mathrm{keV}$ for the $59.5 \mathrm{keV}$ peak position and below $\pm 50 \mathrm{eV}$ for FWHM.

Secondly, the uniformity of the dead layer thickness was measured by observing variation of the peak count rate along the scanning path. The uniformity along the top and side surfaces is confirmed within the limits of the measurements. This method can provide also an additional measure of the absolute thickness of the dead layer. However, the accuracy is much reduced compared to the methods described in section 3.1.2 due to the added uncertainties of the source activity and collimation; the latter being dominant in the order of $10 \%$. 
Table 2. Compilation of selected operational characteristics determined for the prototype detectors including the operational voltage $V_{o p}$ given by the manufacturer, the depletion voltage $V_{d e p}$, dead layer thickness $d_{d}$, active volume fraction calculated from the dead layer $f_{g e o}$ and $f_{a v}$ as determined by the ${ }^{60} \mathrm{Co}$ scan, the active diameter $\emptyset_{a c t}$, and the active height $h_{a c t}$. For comparison the parameters of a reference natural germanium BEGe from the standard Canberra production are shown.

\begin{tabular}{l|ccccccc}
$\begin{array}{l}\text { detector } \\
\text { crystal/slice }\end{array}$ & $\begin{array}{c}V_{o p} \\
{[\mathrm{kV}]}\end{array}$ & $\begin{array}{c}V_{\text {dep }} \\
{[\mathrm{kV}]}\end{array}$ & $\begin{array}{c}d_{d} \\
{[\mathrm{~mm}]}\end{array}$ & $\begin{array}{c}f_{\text {geo }} \\
{\left[\mathrm{m}^{3} / \mathrm{m}^{3}\right]}\end{array}$ & $\begin{array}{c}f_{a v} \\
{\left[\mathrm{~m}^{3} / \mathrm{m}^{3}\right]}\end{array}$ & $\begin{array}{c}\emptyset_{\text {act }} \\
{[\mathrm{mm}]}\end{array}$ & $\begin{array}{c}h_{\text {act }} \\
{[\mathrm{mm}]}\end{array}$ \\
\hline reference & 4.5 & 3.7 & $0.430(23)$ & $0.9534(25)$ & $0.949(18)$ & $80.0(3)$ & $31.0(4)$ \\
1/D & 3.5 & 2.5 & $0.348(15)$ & $0.9530(20)$ & $0.874(13)$ & - & - \\
2/B & 4.5 & 3.5 & $0.761(15)$ & $0.9146(16)$ & $0.911(12)$ & $74.3(6)$ & $29.8(4)$ \\
3/B & 5.0 & 3.7 & $0.43(4)$ & $0.957(4)$ & $0.956(15)$ & $73.5(5)$ & $39.5(4)$ \\
4/C & 4.0 & 3.0 & $0.64(5)$ & $0.931(5)$ & $0.919(13)$ & $72.2(9)$ & $31.8(5)$ \\
4/D & 4.5 & 3.5 & $0.394(12)$ & $0.9544(14)$ & $0.932(15)$ & $72.7(9)$ & $31.7(5)$
\end{tabular}

\subsubsection{Determination of active lengths by surface scans}

The surface scans can be evaluated furthermore in order to extract the active diameter $\emptyset_{a c t}$ at the very top and the active height $h_{a c t}$. From both parameters an independent measure for the active volume $V_{a c t}$ can be derived in principle. However, since some detectors have a conical shape as shown in Fig. [1, the evaluation of $V_{a c t}$ of these detectors can not be accurate. The active lengths are measured by detecting the reduction of the peak count rate at the edges of the active volume in the scans. The obtained values reported in Table 2 have relatively large uncertainties due to the limited positioning accuracy of the scanning device and the relatively large size of the scanning steps.

The intensity distributions $R_{x}$ and $R_{y}$ resulting from the scans on the top of the diode along the $x$ - and $y$-directions, respectively, are compared with MC simulations as shown in the left part of Fig. 2. The rates are low due to collimation and thus multiplied by $10^{5}$ for display. The uniformity of the charge collection along the top is confirmed. As can be noticed, only some minor discrepancies between the simulations and the measurement results were observed, particularly at the edges. These are most likely to be attributed to an imperfect alignment of the scanning device, which was accurate to about $1 \mathrm{~mm}$.

The right part of Fig. 2 displays $R_{z}$ obtained from the scan along the side surface of detector 4/C. The visible variations in count rates are due to blocking of the collimated beam by the copper holder of the detector, which has a non-uniform shape. The top of the detector protrudes by $\sim 1 \mathrm{~mm}$ from the copper holder, around $\mathrm{z}=6 \mathrm{~mm}$. Due to the $\sim 0.5 \mathrm{~mm}$ dead layer, the exposed active part of the detector at this location is significantly smaller than the $\sim 1 \mathrm{~mm}$ diameter of the collimated beam. Therefore the count rate at that position is particularly sensitive to the vertical position of the detector in the holder. Small inaccuracies in the MC model thus lead to the visible discrepancy between the measurements and the MC data.

\subsubsection{Summary of active and dead volume determinations}

The results of these measurements are summarized in Table 6 . The measurements of the geometry 
of the active volume are in general consistent with the expectation that the full volume of the detector is active except for the surface dead layer. Furthermore, the determinations of the dead layer using the comparison of the rate in the collimated scans with MC simulations were also in agreement with the other methods, despite their lower accuracy.

The detector 1/D has been an anomaly in this respect: the measured surface dead layer makes up $(4.7 \pm 0.2) \%$ of the total detector volume while the active fraction $f_{a v}$ measured with ${ }^{60} \mathrm{Co}$ indicates that only $(87 \pm 1) \%$ of the total volume is active; compare also $f_{\text {geo }}$. The deviation could be an indication of a deficiency in charge collection inside the detector volume. Due to the limited accuracy, the scans along detector surfaces were inconclusive with regards to a possible dead layer thickness variation. Nevertheless, this discrepancy is not large enough to pose a serious concern for the detector production for the enriched BEGe detectors of GERDA.

Actually, as a result of these tests the design of the detector holder was changed from the double-ring design to a single ring in order to improve the analysis of the scanning of the enriched BEGes. Also its position with respect to the surface was clearly stated in the specifications.

\subsection{Tests of the spectroscopic performance}

For $0 v \beta \beta$ experiments the energy resolution and the stability of the energy scale are of paramount importance for the identification of a peak that is expected to be rather weak. This peak, if it exists, is more accessible if the background can be reduced, e.g. by PSD. These arguments have motivated the exploration of the BEGe type detectors to substitute the standard semi-coaxial detectors.

The second part of acceptance tests dealed with the characterization of the detector performance. This includes the following measurements of: (i) energy resolution of the $1332.5 \mathrm{keV}$ gamma line of ${ }^{60} \mathrm{Co}$ (see Fig. B), (ii) electronic noise component of the energy resolution (related to the detector capacitance) using a pulser fed to the preamplifier test input, (iii) PSD performance; determined with a ${ }^{228} \mathrm{Th}$ gamma-ray source, using the method described in Ref. [ [ $]$ ] and updated in Ref. [囫 (Fig. 母), (iv) long term stability of the charge collection; determined by periodic measurements with $\gamma$ sources and a pulser over an interval of several months.

The results of this part are summarized in Tables 3 and 4 , while the measurements are described in the following subsections.

\subsubsection{Energy resolution}

The determination of the energy resolution follows the standard prescriptions of spectroscopy, i.e. to accumulate high statistic spectra with the pulser and the ${ }^{60} \mathrm{Co}$ source. The FWHM is the key parameter for the comparison. Fig. 3 shows the zoom onto the peak at $1332.5 \mathrm{keV}$. This range is fitted with a step-like background and a Gaussian resulting in the widths as shown in the plots.

The size of the steps describing the background contributions varies by a factor 2 in these five examples. However, due to different geometries of the detectors an interpretation of this factor is not meaningful, even more not important as in general this background is small, namely on the $\%$-level.

Therefore, the widths of the Gaussian can be extracted rather reliably. For comparison, the five Gaussians are normalized to the same height and are shown in the sixth frame on the right bottom of Fig. 3. The uncertainties due to the fit as shown in the plots are only a minor fraction of the total uncertainty. The latter is dominated by the stability of the energy scale. Therefore, the numbers 

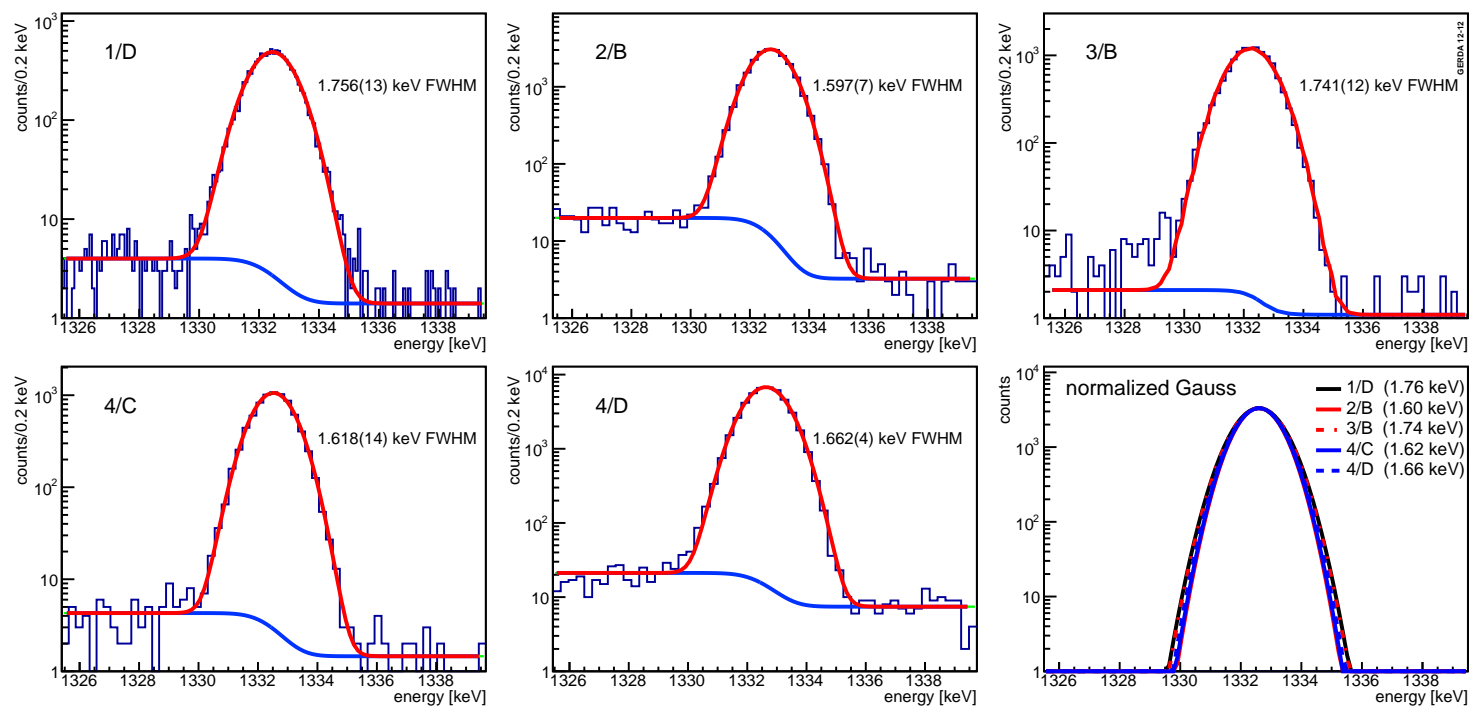

Figure 3. The responses of the five detectors to the $1332.5 \mathrm{keV} \gamma$ line of ${ }^{60} \mathrm{Co}$. The spectra are fitted with a step-like background and a Gaussian with the FWHM as shown in the plots. The bottom right graph shows all Gaussians normalized to the same height for comparison.

given in Table 3 are reduced in the number of significant digits. All detectors except the 1/D exhibit a comparable FWHM for the pulser. Similarly, 1/D has also the worst FWHM for the ${ }^{60}$ Co line.

The worse performance of detector 1/D is not understood. Increased pulser FWHM is an indication of higher electronic noise, which could be e.g. caused by an increased detector capacitance, an effect that possibly is connected with the reduced active volume. However, a possible technical issue with the signal read-out electronics could not be ruled out as a source of this noise. A problem with the charge collection as implicated in section 3.1.6 would in general manifest itself by an appearance of a tail on the side of the $\gamma$ peak; however, this is not observed.

Table 3. The energy resolution of the prototype detectors in comparison to the reference detector and the duration of the long-term stability tests.

\begin{tabular}{l|cc|c}
$\begin{array}{l}\text { detector } \\
\text { crystal/slice }\end{array}$ & $\begin{array}{c}\text { pulser } \\
\text { FWHM [keV] }\end{array}$ & $\begin{array}{c}{ }^{60} \mathrm{Co} \\
\text { FWHM [keV] }\end{array}$ & $\begin{array}{c}\text { long-term test } \\
\text { duration [d] }\end{array}$ \\
\hline reference & 0.49 & 1.63 & 51 \\
1/D & 0.74 & 1.76 & - \\
2/B & 0.45 & 1.60 & 32 \\
3/B & 0.45 & 1.74 & 40 \\
4/C & 0.43 & 1.62 & 85 \\
4/D & 0.44 & 1.66 & 45
\end{tabular}


Table 4. PSD performance of the prototype detectors. To facilitate the comparison among the several detectors, the cut was set to reproduce a $90 \%$ survival probability for the events in the DEP. The uncertainties include statistical as well as systematic contributions. The energies of the DEP, SEP, and FEP lines are given in $\mathrm{keV}$ together with the Co summation peak (SP).

\begin{tabular}{l|ccccc}
\multirow{2}{*}{$\begin{array}{l}\text { detector } \\
\text { [crystal/slice] }\end{array}$} & \multicolumn{5}{|c}{ surviving fraction after PSD analysis [\%] } \\
\cline { 2 - 6 } & DEP & FEP1 & SEP & FEP2 & ${ }^{60}$ Co SP \\
& 1592.3 & 1620.6 & 2103.5 & 2614.5 & 2505.7 \\
\hline reference & 90 & $10.4 \pm 0.9$ & $5.3 \pm 0.5$ & $7.7 \pm 0.4$ & $0.28 \pm 0.02$ \\
1/D & 90 & $14.3 \pm 0.5$ & $8.7 \pm 0.5$ & $13.1 \pm 0.5$ &.-- \\
2/B & 90 & $10.3 \pm 1.4$ & $5.0 \pm 0.6$ & $7.4 \pm 0.6$ & $0.093 \pm 0.011$ \\
3/B & 90 & $7.6 \pm 0.7$ & $4.3 \pm 0.4$ & $5.7 \pm 0.5$ & $0.090 \pm 0.012$ \\
4/C & 90 & $9.0 \pm 0.3$ & $4.1 \pm 0.3$ & $6.1 \pm 0.5$ & $0.068 \pm 0.011$ \\
4/D & 90 & $8.5 \pm 0.6$ & $4.7 \pm 0.5$ & $6.4 \pm 0.5$ & $0.088 \pm 0.009$
\end{tabular}

\subsubsection{Pulse shape discrimination}

The two $\beta$ s from the $0 v \beta \beta$ event have a rather short path length in the germanium detector depositing their energy in a rather small volume of a few $\mathrm{mm}^{3}$ ('single-site'). In contrast, photons of equivalent energy will undergo several scatterings requiring a significantly larger volume ('multisite'). This behavior will be reflected in the time structure of the charge signal. To investigate the effect and to define analysis cuts one has to retire to $\gamma$ sources of higher energy, e.g. to ${ }^{228} \mathrm{Th}$ with its $2614.5 \mathrm{keV}$ quanta. The double escape peak (DEP) is assumed to exhibit the same behavior as the $0 v \beta \beta$ event. The kinetic energy of the electron-positron pair is deposited in a similarly small volume while the two annihilation quanta escape the active volume of the detector. In contrast, for the full energy peak (FEP) typically involves events with multiple spatially-separated interactions, either by Compton scatterings followed by photo-absorption, or at high energies also via pair production and subsequent absorption of both annihilation photons. For detailed explanations and their validation see Refs. [8, 9].

The ${ }^{228} \mathrm{Th}$ source is rather convenient as it provides a $1592.5 \mathrm{keV}$ line, which is the DEP of the $2614.5 \mathrm{keV}$ line in ${ }^{208} \mathrm{Tl}$. In its vicinity, the FEP of the $1620.6 \mathrm{keV} \gamma$ of ${ }^{212} \mathrm{Bi}$ appears. Both isotopes are ${ }^{228} \mathrm{Th}$ progenies. Furthermore, both are relatively close to the $Q_{\beta \beta}$ value of $2039 \mathrm{keV}$.

The ${ }^{228} \mathrm{Th}$ spectra taken with the five detectors are shown in Fig. 4 for the region of the DEP and FEP as discussed above. The original spectra are shown as red histograms. Applying the PSD cut after optimization and normalizing to a survival fraction of $90 \%$ for the DEP the blue histograms are obtained. In order to appreciate the overall effect of PSD a large range from 600 to $3000 \mathrm{keV}$ is plotted in the frame at the bottom right of Fig. [.

The effect of PSD is seen clearly in the reduction of the FEP line at $1620.6 \mathrm{keV}$ for all detectors. The relevant fractions are compiled in Tab. $\$$ where FEP1 corresponds to the discussed line at $1620.6 \mathrm{keV}$. Except for the 1/D detector the reduction of the 'multi-site' events within the peaks to $8-10 \%$ is reachable. The uncertainties include statistical as well as systematic contributions, the latter are derived from previous studies as described in Ref. [8, Q]. The performance is quite 

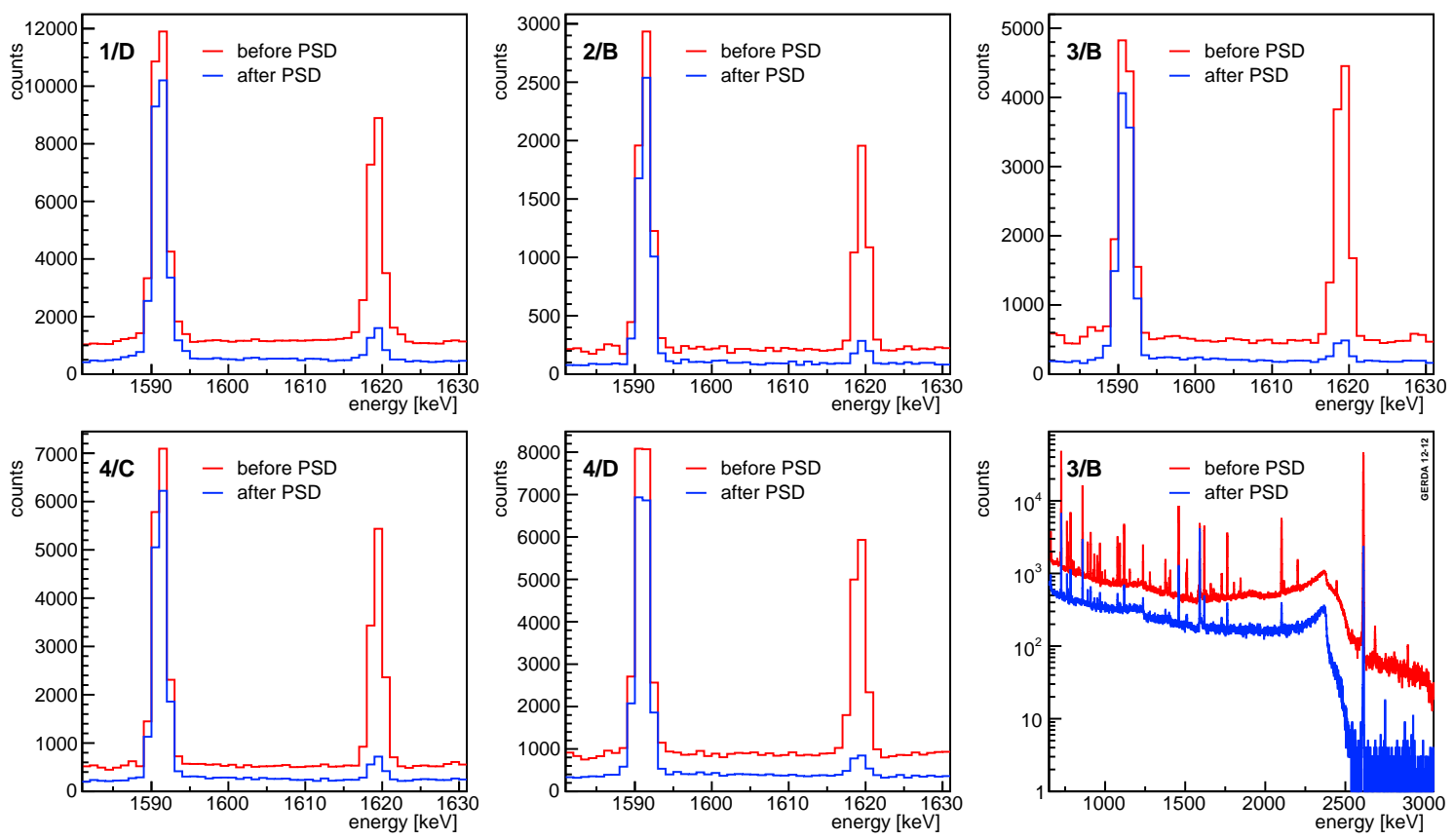

Figure 4. Spectra of ${ }^{228} \mathrm{Th}$ taken with the five detectors tested, zoomed into the range of the DEP at $1592.3 \mathrm{keV}$ and the FEP at $1620.6 \mathrm{keV}$ (see text). The standard spectra are given in red (upper curves); applying the PSD cut reduces the data as shown in blue (lower curves). The overall effect of the PSD cut is demonstrated by example of detector 3/B for the range from 650 to $3000 \mathrm{keV}$ (bottom, right, $\log$ scale).

similar for all other detectors.

The bottom right panel in Fig. - 4 showing the complete spectrum above $650 \mathrm{keV}$ demonstrates the effect of the PSD cut for a wide energy range. Actually, it hints at the fraction of 'multi-site' events contributing to the total spectrum. The separation of SSE and MSE is energy independent which was validated in the range from 1.4 to $2.4 \mathrm{MeV}$, interesting for $0 v \beta \beta$ decay. The reduction is more or less constant at $30 \%$ up to the Compton edge around $2300 \mathrm{keV}$, only above that energy the intensity is reduced to about $5 \%$ by the PSD cut due to the prevalence of MSE.

Additional information is provided for the single escape peak (SEP) at $2103.5 \mathrm{keV}$ and the $2614.5 \mathrm{keV}$ line of ${ }^{208} \mathrm{Tl}$ (FEP2). The last column of Tab. 7 shows the PSD performance for the summation peak (SP) of the two ${ }^{60} \mathrm{Co}$ lines that is seen at an energy of $2505.7 \mathrm{keV}$. The summation effect results in highly 'multi-site' events, responsible for more than two orders of magnitude stronger suppression than is reached in the FEP and SEP. The suppression achieved with the four tested detectors is in this case much better than that quoted for the reference BEGe, as the analysis procedure has ben improved meanwhile.

The PSD performance of the detector 1/D was again anomalous with higher than typical background survival fractions. The cause could possibly be due to the higher electronic noise present in the measurements of this detector, or due to less favorable electric field distribution inside the detector, possibly related to the charge collection deficiency indicated in section 3.1.6. 


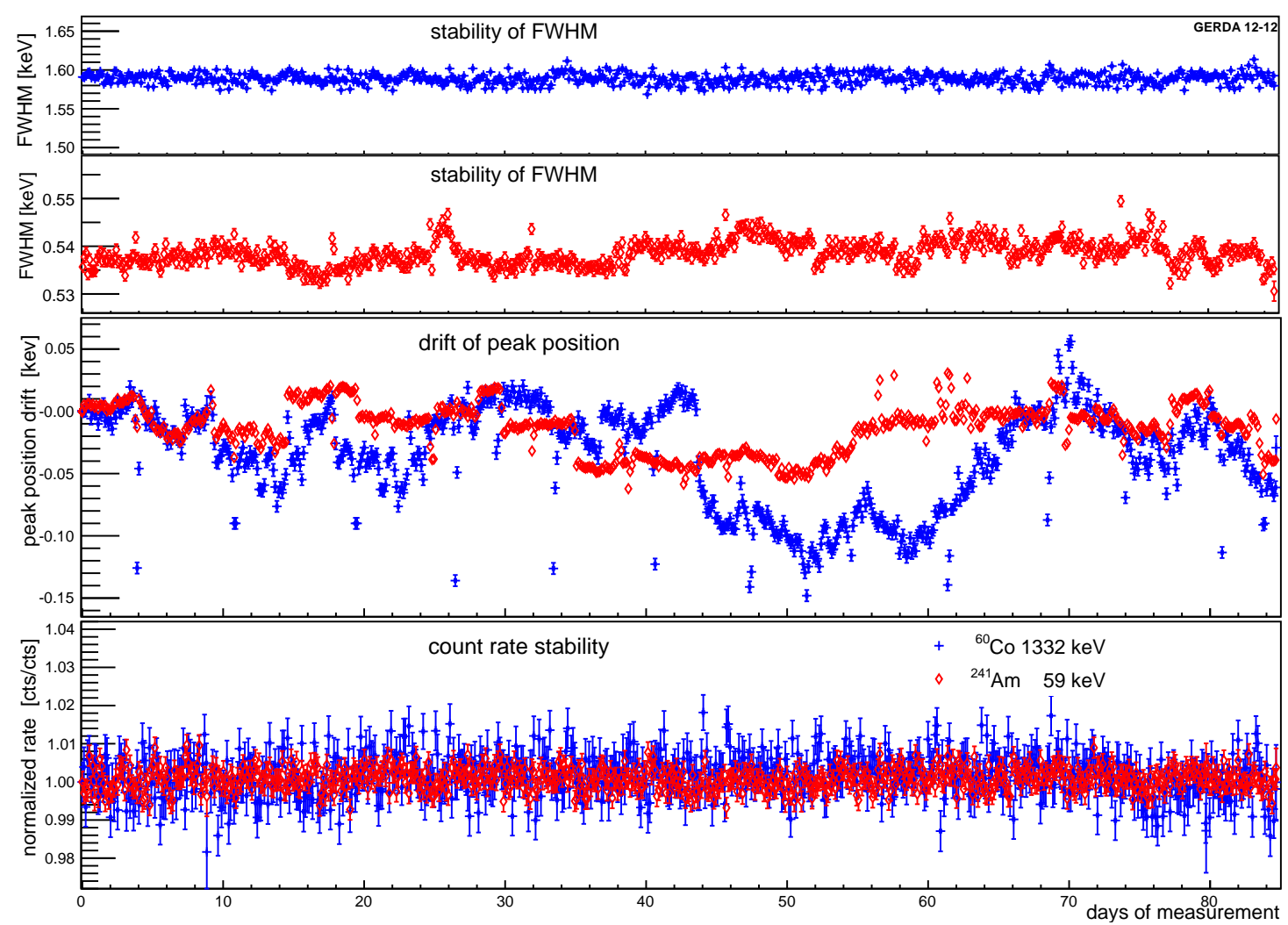

Figure 5. Long term stability test of detector $4 / \mathrm{C}$ with two sources. The blue crosses represent the ${ }^{60} \mathrm{Co}$ data whereas the red diamonds show the results for ${ }^{241} \mathrm{Am}$.

\subsubsection{Long-term stability}

The last column of Table B gives the duration of the long term tests lasting between 1 and 3 months. The diodes were irradiated alternating with an ${ }^{241} \mathrm{Am}$ and a ${ }^{60} \mathrm{Co}$ source. The count rates were normalized to the start rate and plotted in Fig. 5 together with the stability of peak position and energy FWHM. The count rate stays well within $\pm 1 \%$. Measurement of possible charge collection effects on peak position variation was limited by the inherent variability of the DAQ system gain (characterized via shorter pulser stability measurements) to the upper limit of about $\pm 0.01 \%( \pm 0.1 \mathrm{keV})$ at the $1332.5 \mathrm{keV}$ line and about $\pm 0.05 \%( \pm 0.05 \mathrm{keV})$ at the $59.5 \mathrm{keV}$ line. The FWHM remained also very stable within approximately $\pm 20 \mathrm{eV}$. Similar results were obtained with the other detectors, except of 1/D, for which a long term test was not performed.

\section{Conclusions}

The whole production chain, from material procurement to detector operation, was successfully tested with depleted germanium left over after the enrichment process. Various detectors were produced with modifications of the production processes with the purpose to maximize the yield of the upcoming new BEGe detectors from the available enriched Ge. The latter, after reduction and purification at PPM Pure Metals, amounts to $35.4 \mathrm{~kg}$ plus a $1.1 \mathrm{~kg}$ tail of slightly lower than 
$6 \mathrm{~N}$ quality which can be recycled in a subsequent production run. The production of new BEGe detectors for the second phase of GERDA and their characterization is ongoing.

The measured characteristics and performance of the tested depleted detectors were as good as those of a reference BEGe from standard Canberra production made of natural germanium. This proves that high-purity germanium material crystals produced from isotopic modified germanium raw material according to the described production steps have the same properties as those made from standard material and processing by Canberra. The depleted germanium has the same chemical history as the enriched germanium for the second phase of GERDA. Thus, this test confirms the viability of the production chain for enriched BEGe detector production.

Good-performance detectors were produced successfully from germanium crystal slices which are normally discarded before BEGe production. In addition, the surfaces of some slices were machined less than usual with no loss of performance. Thus, the smaller material losses will increase the yield of total detector mass from the limited available enriched germanium. Presently, the yield of detector mass was about $50 \%$ with respect to the $6 \mathrm{~N}$ starting material. It can be further augmented by increasing the initially available enriched germanium, as in the case of the subsequent enriched germanium production. The residual material, including seed and tail ends of crystals, as well as kerf and grinding losses can be recovered and chemically reprocessed, and a large fraction can return into the crystal production process.

The viability of the production processes of isotopically modified BEGe detectors for the $0 v \beta \beta$ decay experiment GERDA was proven within a rather short period of less than 2 years including all steps. The vital part for the enriched program amounted to about $60 \%$ of this time.

A precise positioning system for the radioactive sources with well known intensity is of paramount importance for the characterization process of new detectors. Such a system was established for the tests of the enriched detectors. For a $0 v \beta \beta$ experiment the precision of the active volume is the parameter of highest relevance followed by the energy resolution itself.

\section{Acknowledgments}

This work is supported financially by the German Federal Ministry for Education and Research (BMBF), the German Research Foundation (DFG) via the Excellence Cluster Universe, the Italian Istituto Nazionale di Fisica Nucleare (INFN), the Max Planck Society (MPG), the Swiss National Science Foundation (SNF). The institutions acknowledge also internal financial support.

The authors acknowledge the excellent cooperation with Canberra Oak Ridge, Canberra Meriden, and Canberra Olen.

We thank Thomas Kihm for the DAQ software [19] and Matthias Laubenstein for valuable help.

\section{References}

[1] Letter of Intent to LNGS, see also: hep-ex/04040390, GERDA Proposal to LNGS (2004), http://www.mpi-hd.mpg.de/gerda/

[2] K.-H. Ackermann et al., The Gerda experiment for the search of $0 v \beta \beta$ decay in ${ }^{76} G e$, Eur. Physics J. C 73 (2013) 2330. 
[3] M. Gunther et al., Heidelberg-Moscow $\beta \beta$ experiment with ${ }^{76}$ Ge: full setup with five detectors, Phys. Rev. D55 (1997) 54.

[4] C.E. Aalseth et al., IGEX ${ }^{76}$ Ge neutrinoless double-beta decay experiment: Prospects for next generation experiments, Phys. Rev. D 65 (2002) 092007.

[5] H.V. Klapdor-Kleingrothaus et al., Search for neutrinoless double beta decay with enriched ${ }^{76} \mathrm{Ge}$ in Gran Sasso 1990-2003, Phys. Lett. B586 (2004) 198.

[6] Canberra Semiconductor NV, Lammerdries-Oost 25, B-2430 Olen, Belgium.

[7] M. Barnabé Heider, D. Budjáš, K. Gusev and S. Schönert, Operation and performance of a bare broad-energy germanium detector in liquid argon, JINST 5 (2010) P10007.

[8] D. Budjáš, M. Barnabé Heider, O. Chkvorets, N. Khanbekov and S. Schönert, Pulse shape discrimination studies with a broad-energy germanium detector for signal identification and background suppression in the GERDA double beta decay experiment, JINST 4 (2009) P10007.

[9] M. Agostini, C. A. Ur, D. Budjáš, E. Bellotti, R. Brugnera, C. M. Cattadori, A. di Vacri, A. Garfagnini, L. Pandola and S. Schönert, Signal modeling of high-purity Ge detectors with a small read-out electrode and application to neutrinoless double beta decay search in Ge-76, JINST 6 (2011) P03005.

[10] Currently known as Joint Stock Company "Production Association Electrochemical Plant" (JSC "PA Electrochemical Plant"), uranium enrichment enterprise of the State Atomic Energy Corporation "Rosatom".

[11] PPM Pure Metals, GmbH, in Langelsheim, Germany.

[12] Canberra Oak Ridge, USA.

[13] Canberra Broad Energy Germanium (BEGe) detector, http://www. canberra.com/products/485.asp.

[14] M. Agostini, L. Pandola, P. Zavarise and O. Volynets, GELATIO: a general framework for modular digital analysis of high-purity Ge detector signals, JINST 6 (2011) P08013.

[15] M. Tarka, Studies of Neutron Flux Suppression from a $\gamma$-ray Source and The GERDA Calibration System, Dissertation, University of Zürich (2012).

[16] D. Budjáš, Germanium detector studies in the framework of the GERDA experiment, Dissertation, University of Heidelberg (2009).

[17] D. Budjáš, M. Heisel, W. Maneschg and H. Simgen, Optimisation of the MC-model of a p-type Ge-spectrometer for the purpose of efficiency determination, Appl. Radiat. Isot. 67 (2009) 706.

[18] M. Agostini, E. Bellotti, R. Brugnera, C. M. Cattadori, A. D'Andragora, A. di Vacri, A. Garfagnini, M. Laubenstein, L. Pandola, C. A. Ur, Characterization of a broad energy germanium detector and application to neutrinoless double beta decay search in ${ }^{76} \mathrm{Ge}$, JINST 6 (2011) P04005.

[19] MIZZI Computer Software GmbH, www .mizzi-computer. de. 